\section{Formation of an Aortic Graft-Enteric Fistula 20 Years after Aortic Graft Placement}

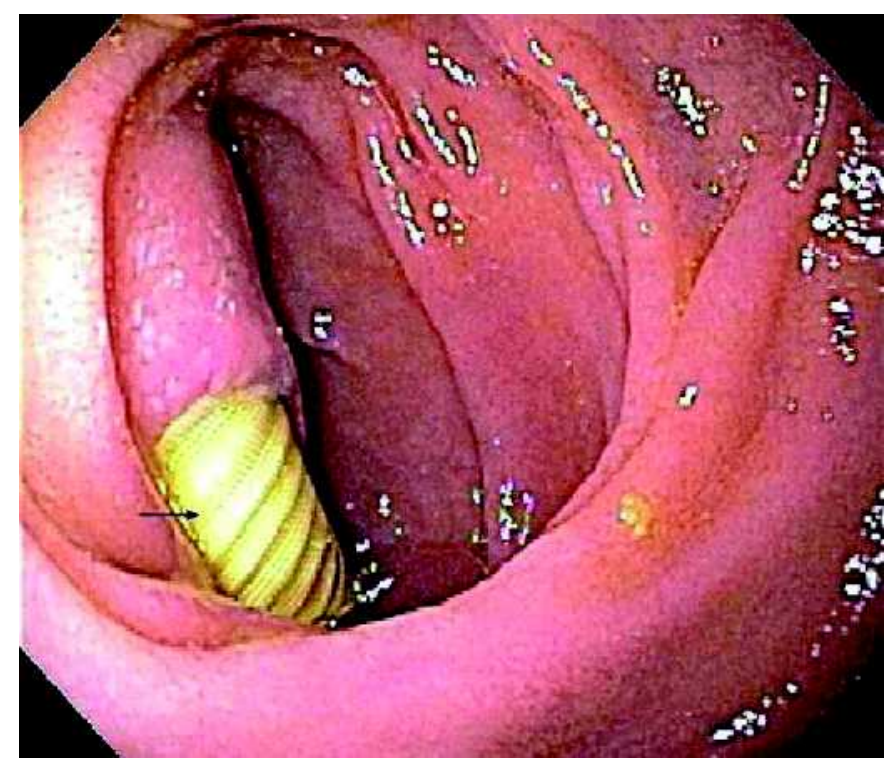

Figure 1 An 80-year-old man presented with epigastric discomfort, melena, fever, and chills. He had a history of an aorto-bifemoral graft, placed for the treatment of claudication about 20 years prior to this presentation. Computed tomography of the abdomen revealed an air bubble in the graft and a small fluid collection around the graft. Blood cultures were positive for bacteremia and fungemia. Upper endoscopy was per-

formed and the appearance was diagnostic of an aorto-duodenal fistula. A large area of ulceration which exposed a section of Dacron graft (arrow) was identified in the third portion of the duodenum. There was no evidence of active bleeding. The patient underwent excision of the infected aortic graft and repair of the aorto-enteric fistula, and was doing well at his 6-month follow-up.

\section{T. E. Yusuf, J. E. Clain}

Division of Gastroenterology and Hepatology, Mayo Clinic, Rochester, Minnesota, USA

\section{Corresponding Author}

\section{T. E. Yusuf, M.D.}

Division of Gastroenterology and Hepatology

Mayo Clinic 200 First Street sw

Rochester, Minnesota 55905 USA

Fax: +1-507-266-3939

E-mail: yusuf.tony@mayo.edu 